\title{
Nonperturbative theory and computation of the nonlinear response of He to dc and ac fields
}

\author{
Theodoros Mercouris, ${ }^{1,}$ Spyros I. Themelis, ${ }^{1, \dagger}$ and Cleanthes A. Nicolaides ${ }^{1,2, \uparrow}$ \\ ${ }^{1}$ Theoretical and Physical Chemistry Institute, National Hellenic Research Foundation, 48 Vas. Constantinou Ave., \\ 11635 Athens, Greece \\ ${ }^{2}$ Physics Department, National Technical University, Athens, Greece \\ (Received 20 July 1999; published 13 December 1999)
}

\begin{abstract}
We have nonperturbatively computed dc-field tunneling rates, linear and nonlinear dynamic polarizabilities, and two-, three-, four-, and five-photon ionization rates for $\mathrm{He}$, for frequencies $\omega$ in the range $\hbar \omega=4.9-26.4 \mathrm{eV}$ and for intensities in the range $3.5 \times 10^{12}-1.715 \times 10^{14} \mathrm{~W} / \mathrm{cm}^{2}$. The calculations systematically incorporated electronic structure and electron correlation effects in the discrete and continuous spectra, while the twoelectron state symmetries were ${ }^{1} S,{ }^{1} P,{ }^{1} D,{ }^{1} F,{ }^{1} G$, and ${ }^{1} H$ of even and odd parities. The calculations were made by implementing a time-independent many-electron, many-photon theory which obtains cycle-averaged energy shifts $\Delta(\omega, F)$ and energy widths $\Gamma(\omega, F)$ nonperturbatively, where $F$ is the field strength. These quantities are the real and imaginary parts of a complex eigenvalue $z_{0}$ (the result of the overall calculation), after the subtraction of the unperturbed real energy $E_{0}$.
\end{abstract}

PACS number(s): 32.80.Rm, 32.80.Fb, 32.10.Dk, 42.65.An

\section{INTRODUCTION}

In this paper we consider the many-electron problem of $a b$ initio calculating the response (linear or nonlinear) of an atomic state to an external dc or ac field in the dipole approximation. The formalism is time independent. The corresponding form of the Hamiltonian is known (see Sec. II), while the observables are averages over an optical cycle and depend on the strength, frequency, and polarization of the field, which is supposed to have been turned on adiabatically and to interact with the atom at constant intensity. The atomfield interaction causes the mixing of atomic states, with concomitant observable phenomena and properties associated with energy shifts and transitions to, from, and within the discrete and continuous spectra. The number of publications on the theory of such properties is large, ranging from pure formalism and phenomenology to calculations based on models, or on empirical imput or on first principles, without or with significant approximations, depending on the number of active electrons (one or many) and on the order of perturbation theory with which a property is connected, or which is deemed necessary by the strength of the interaction. For reviews the reader is referred to [1-8]. The terms in the atomfield Hamiltonian describing the interactions (nonrelativistic or relativistic) which characterize quantitatively the measured quantities can be classified as follows: $(A)$ The oneelectron atomic structure operators. The strength parameter is that of the nuclear charge. $(F)$ The one-electron atom-field interaction operators. The strength parameter for the fixed photon frequency is the field strength. $(C)$ The two-electron atomic structure operators. (Coulomb or Breit-Pauli.) For $\mathrm{N}$-electron atomic bound, autoionizing, and scattering states of the Hamiltonian composed of $A$ and $C$, computing their wave functions and properties constitutes the essence of the

\footnotetext{
*Electronic address: thmerc@eie.gr

†Electronic address: stheme@eie.gr

*Electronic address: can@eie.gr
}

many-electron problem. When $F$ is added to such a state, a new and more complex problem arises, in general; the timeindependent many-electron, many-photon (MEMP) problem. Its quantitative solution allows a determination of cycleaveraged observables, such as energy shifts (ES's), rates of tunneling $(T)$ and partial and total rates of multiphoton ionization (MPI), which are calculated in this paper.

The computational and interpretative challenge of the MEMP problem is created by two factors: (1) the diversity and complexity of the structures of the discrete states and of the multichannel continua (with resonances); and (2) their interplay with the field characteristics, especially for strong fields. One drastic reduction of the related formal and computational difficulties is achieved by resorting to modelseven one dimensional. However, given the now available computer power, it is much more appropriate to treat the physics of $\mathrm{N}$-electron atoms and molecules in terms of the $N$-electron Hamiltonian and wave functions, whereupon reductions can be made depending on the state(s) and property under consideration. The prerequisites for a reliable quantitative treatment include the capacity of theory to implement in a tractable way advanced methods of computing electronic structure and scattering, just as this is necessary with ordinary spectroscopic properties (e.g., one-photon absorption oscillator strengths or fine and hyperfine structure).

As regards (2), it is customary to distinguish two regimes: the weak and strong-field regimes. A rough distinction is obtained by comparing the field-strength (in a.u.) with the binding energy (in a.u.) of the outer electron(s) which are active during the response of the state to the external field. When the field strength is orders of magnitude smaller, one may expect to deal with a weak-field regime, a situation which is formally understood in terms of the expressions of lowest-order perturbation theory (LOPT) in the series expansion of the field strength. This last statement may serve as a definition of the weak field for the quantitative interpretation of a quantity associated with an $n$-photon process: when the measured (meaning accurate) quantity is produced exactly by the corresponding expression from LOPT, (assuming the cal- 
culation can be done), the weak-field regime holds. However, even in this case, the many-electron aspects introduced by $C$, and the requirement of computing explicitly (sum over states) or implicitly (inhomogeneous differential equations) the contribution of the complete set of states of the discrete and the continuous spectra, makes the calculation of nonlinear ionization rates and polarizabilities for polyelectronic states quite cumbersome even at the LOPT level, especially when the desired order of perturbation is high.

In support of the above comment, we refer to Refs. [1-8] and [9-13] for work on the ground and excited states of the $H$ atom and to Refs. [14], [15] for work on the He ground state. For the latter, which is the state investigated in this paper, Liu's [15] recent LOPT calculations up to the 15th order have provided benchmark data for frequencydependent nonlinear susceptibilities at four frequency values.

If the measured (accurate) quantity does not agree with that which is computed from the LOPT formulation, the field can be defined as strong. This implies that higher-order terms contribute and that there is no exact equivalence between the order of the physical process (e.g., $n$-photon absorption) and the LOPT expression. In principle, there are two possible remedies. One is to compute terms beyond LOPT, until good convergence is obtained. This has not yet been done for a polyelectronic state. The other is to design the whole calculation within a nonperturbative (variational) framework, where ES's and the rates of $T$ and MPI are computed to all orders. In this case, provided the formalism is suitable and generally applicable, the accuracy of the calculation is subject only to the quality and the physical relevance of the function spaces used to represent the states of the discrete and the continuous spectra when calculating the matrix elements involving operators $A, F$, and $C$.

The work presented here deals with the theory and computation of strong field properties of $\mathrm{He}^{1} S$. It was carried out by implementing the nonperturbative MEMP theory (MEMPT) that was formulated in the mid-1980s [16-20]. The end result of the computation using the MEMPT is a complex eigenvalue whose real part is connected directly to the field-induced energy shift and to concomitant linear and nonlinear polarizabilities, and whose imaginary part is the cycle-averaged rate of the ionization of the state under investigation. This approach, which is explained in Sec. II, was first applied to the $\mathrm{H}^{-}$and $\mathrm{Li}^{-}$ground states [16-20], which were treated as many-electron systems and not as oneelectron models. A number of conclusions were reached following the analysis of the results. For example, it was reported for the first time that the then existing qualitative discrepancy between calculations based on models of the negative ions and on $a b$ initio theory should be resolved in favor of calculations using models, and that much of the essential physics of negative ions in strong ac and dc fields is caused by final-state effects $[17,18]$. In the case of low-lying excitation of $\mathrm{Li}^{-}$studied for the first time in Ref. [17], (up to and including $\operatorname{Li} 1 s^{2} 2 p^{2} P^{o}$ threshold), these effects were identified as "even or odd shape resonances, interchannel mixing and field-perturbed free electron orbitals," (Ref. [17], p. 49). Further analysis of the effects on MPI rates in $\mathrm{Li}^{-}$from the interplay between field intensity and electronic structure and interchannel coupling were later reported from calculations based on the time-dependent Schrödinger equation [21].

In addition to calculations and analyses of MPI rates without and with the presence of a dc field, and of dynamic linear and nonlinear polarizabilities of the ground states of $\mathrm{H}^{-}$and $\mathrm{Li}^{-}$[16-20], applications of the MEMPT with correlated wave functions have demonstrated the effects of abovethreshold detachment without and with a dc field [22], of the polarization of doubly excited states (DES's) [23], of transfer of the frequency-dependent polarization, of the autoionization rate and of stabilization through state mixing in DES's $[23,24]$ and of magnetic fields interacting with DES's [25]. Furthermore, fundamental aspects of this theory were also adopted to the formulation of a quantum-mechanical approach to the calculation of pair correlations in the continuous spectrum, in connection with the problem of computing single or MPI rates of double-electron ejection at and near the ionization threshold [26].

Here it is to be noted that in more recent years the incorporation of electron correlation and atomic structure into nonperturbative, time-independent calculations of rates of MPI of the ground states of $\mathrm{H}^{-}$and of $\mathrm{Li}^{-}$was also achieved by the collaboration of a few groups [27-29] via a Floquet formalism combining the complex coordinate rotation (CCR) method (see Sec. II) with $R$-matrix scattering theory for the treatment of the asymptotic functions. The same approach was applied by Purvis et al. [27] to the calculation of MPI rates in He, quantities which are also calculated here, albeit for a different and broader combination of frequencies and intensities.

In many cases, the results of state-specific MEMPT calculations on field-induced properties of $\mathrm{H}^{-}, \mathrm{He}$, and $\mathrm{Li}^{-}$are verified quantitatively by conventional calculations of much larger scale. For example, consider the dynamic polarizability $\alpha(\omega)$, of $\mathrm{H}^{-}$. Kutzner, Felton, and Winn [30(a)] used the accurate photoabsorption cross section previously obtained by Geltman and the dispersion relation between the real and imaginary parts of $\alpha(\omega)$ to compute $\alpha(\omega)$ (real) for $\omega$ below as well as above threshold. The agreement between the MEMPT results [20] and those of Ref. [30(a)] is very good over the whole range of $\omega$ values [see Fig. 1 of Ref. [30(a)]]. Comparisons can also be made for the static hyperpolarizabilities $\gamma$. For He, Nicolaides and Themelis [31] reported $\gamma=42.78$ a.u. The earlier full configuration interaction (CI) result of Jaszunski and Roos [32] is 43.7 a.u., while the LOPT calculation with a large number of $r_{12}$ basis sets by Bishop and Pipin [14(b)] gave 43.104 a.u. An earlier such calculation by Bishop and Lam [14(a)], where $p p$-type configurations had not been included, gave 41.90 a.u. For $\mathrm{H}^{-}$, the authors of Ref. [20] obtained $\gamma=7.6 \times 10^{7}$ a.u., a value which was confirmed within 5\% by Pipin and Bishop [30(b)] who obtained $8.02 \times 10^{7}$ a.u. Finally, for $\mathrm{Li}^{-}$, the authors of Ref. [19] reported $\gamma=5.1 \times 10^{8}$ a.u., while at the same time Archibong and Thakkar [33] obtained $12.7 \times 10^{8}$ a.u. from a coupled-cluster calculation. A more recent calculation by Sauer [34] at the full CI level gave $5.65 \times 10^{8}$ a.u. in very good agreement with the MEMPT result.

Although the $a b$ initio treatment of a state of an atomic 
negative ion requires a careful consideration of the details of the radials of the zero order and of the correlation functions when computing nonlinear response properties, the computational advantage from the point of view of economy is the fact that they lack an intermediate discrete spectrum. It is for this reason that only $\mathrm{H}^{-}$and $\mathrm{Li}^{-}$were chosen by us initially, at a time when our computational facilities were limited. In the meantime, much better such facilities have become available and calculations on neutral ground and singly and doubly excited states have been carried out regarding dc-fieldinduced $T$ rates and static linear and nonlinear polarizabilities e.g., (see, Refs. [30, 35-38]).

In this paper, we present results of an extensive study of the response of neutral $\mathrm{He}^{1} S$ to weak and strong dc and ac fields over a broad range of intensities and photon frequencies below and above the ionization threshold. Specifically, for the dc-field case the strength range is $0.06-0.20$ a.u. For the ac field, the intensity ranges from $3.5 \times 10^{12}$ to 1.715 $\times 10^{14} \mathrm{~W} / \mathrm{cm}^{2}$, and the photon frequencies range from 4.9 to $26.4 \mathrm{eV}$.

The paper is organized as follows. In Sec. II we discuss the background and methodology of the MEMPT. In Sec. III we present our results for the nonlinear response of $\mathrm{He}$ to strong external $\mathrm{dc}$ and ac fields. Tunneling and MPI rates are provided for intensities up to $1.715 \times 10^{14} \mathrm{~W} / \mathrm{cm}^{2}$, while using the results on ES's for low strengths, linear and nonlinear dynamic polarizabilities are obtained. Section IV summarizes the essence of the MEMPT and its present application to He.

\section{THEORY}

\section{A. General}

Assuming that the external ac field is turned on adiabatically with respect to the atomic state with which it interacts, the fact that it is periodic with time allows a formal description of its interaction with the atom to be done in a timeindependent framework, whereby the computed properties are averages over a field cycle [2,3,39-41]. This description goes under the generic name "Floquet theory." In this case, the total Hamiltonian for linearly polarized monochromatic light along the $z$ axis in the dipole approximation with frequency $\omega$ is given by

$$
H_{\mathrm{ac}}=H_{\mathrm{atom}}+\hbar \omega \alpha_{\omega}^{\dagger} \alpha_{\omega}-\frac{1}{2} F_{\mathrm{ac}} z\left(\alpha_{\omega}^{\dagger}+\alpha_{\omega}\right),
$$

where $\alpha_{\omega}^{\dagger}\left(\alpha_{\omega}\right)$ are the photon creation (annihilation) operators, and $F_{\mathrm{ac}}$ is the field strength in a.u. ( 1 a.u. $=5.14$ $\times 10^{9} \mathrm{~V} / \mathrm{cm}$ ). In the case of the dc field, the total Hamiltonian is

$$
H_{\mathrm{dc}}=H_{\mathrm{atom}}-\frac{1}{2} F_{\mathrm{dc}} z,
$$

where $F_{\mathrm{dc}}$ is the field strength in a.u.

Given the fact that the stationary state spectrum of $H_{\text {Атом }}$ contains square-integrable $\left(\mathcal{L}^{2}\right)$ discrete as well as energynormalized scattering states, it is clear that a rigorous treatment of the atom+field system, whose Hamiltonians are given by Eqs. (1) and (2), involves the continuous spectrum as a function of field strength. As is well known, its explicit consideration complicates theory as well as computation. A significant practical step in the possibility of including the contribution of the continuous spectrum in a nonperturbative calculation of field-induced ionization rates was made in $1976-1978$ by Reinhardt and co-workers $[42,43]$. They used $H_{\mathrm{dc}}$ [Eq. (2)] for the $1 s$ state of $H$, and applied the procedure of CCR developed earlier by Doolen and co-workers [44,45] for the calculation of atomic resonance states. Reinhardt and co-workers empirically found that the complex pole corresponding to the dc-field-induced resonance was identifiable, although the original mathematical theory of Aquilar, Balslev, and Combes [46] for the spectrum of the rotated Coulomb Hamiltonian, $H(\theta), r \rightarrow r e^{i \theta}$, did not apply. We recall that the full spectrum of $H(\theta)$, including possible resonances on the second Riemann sheet, is revealed, although only $\mathcal{L}^{2}$ basis sets and no energy-normalized scattering functions are employed. [The applicability of coordinate transformations (rotation or translation) in the case of complex energy resonances induced by electric fields has been explained from the point of view of regularization of the resonance eigenfunction [47].] At the same time, Chu and Reinhardt [40], and later Maquet, Chu, and Reinhardt [41], noting the similarity between the Hamiltonians (1) and (2), extended Floquet theory to include the continuous spectrum by using the rotated Hamiltonian $H_{\mathrm{ac}}(\theta)$ for hydrogen. From the calculated complex eigenvalues of $H_{\mathrm{ac}}(\theta)$, which was diagonalized on a large basis of generalized orthonormal Laguerre functions, they obtained frequency-dependent oneand two- photon ionization rates for $F_{\mathrm{ac}}$ ranging from $10^{-4}$ to 0.20 a.u., and presented a thorough analysis of the method and its results. (For extensive discussion and applications of the CCR Floquet theory, see the reviews by Chu [48] and Potvliege and Shakeshaft [49].)

In the CCR Floquet method, the coordinate transformation $r \rightarrow \rho=r e^{i \theta}$ is introduced in the Hamiltonians $H_{\mathrm{dc}}$ and $H_{\text {ac }}$, which are then diagonalized on a large, single basis set of $\mathcal{L}^{2}$ functions. The method searches for the proper complex eigenvalue, whose imaginary part gives the rate of fieldinduced ionization (FII). However, brute-force diagonalization of Hamiltonian matrices on $N$-electron function spaces made up from a single one-electron basis set is very uneconomical even for the calculation of wave functions and energies of free atoms, especially in correlated excited states. The situation becomes much worse, not only for obtaining results but also for understanding them, if one wishes to diagonalize $H_{\mathrm{dc}}(\theta)$ and especially $H_{\mathrm{ac}}(\theta)$ in this way for $\mathrm{N}$-electron states, as a function of frequency and intensity. The aforementioned bottleneck in dealing with polyelectronic states, which essentially characterizes any many-body problem for which the adopted approach depends on the diagonalization of large matrices constructed from single basis sets, is bypassed in the approach to the MEMP problem, which is described in Sec. II B.

\section{B. Many-electron, many-photon theory}

FII in the time-independent picture can be conceptualized in terms of field-dressed states interacting with the con- 
tinuum, in analogy with autoionization [50,51]. Accordingly, the framework is that of one or more discrete states mixing with one or more continua (open channels). In the case of autoionization, the mixing is due to an effective interaction intrinsic to the atomic (molecular) Hamiltonian, whereas in the case of FII it is due to the $H_{\mathrm{dc}}$ and $H_{\mathrm{ac}}$. The type, number, and magnitude of interaction matrix elements involving operators $A, F$, and $C$ and containing the physics of this problem, depend on the initial bare state, on the spectrum of the system under consideration, and on the frequency, polarization, and intensity of the external field.

As regards autoionization, a series of papers in the late 1970s [52-54] provided the basis for the many-electron analysis of resonances and for their computation in terms of separately optimized functions spaces, reflecting the physics of decay. The corresponding fundamental equation is the complex eigenvalue Schrödinger equation (CESE)

$$
\left(H-z_{0}\right) \Psi\left(\mathbf{r}, z_{0}\right)=0,
$$

where $z_{0}$ is complex, $z_{0}=E-i \Gamma / 2$, and $\Psi\left(\mathbf{r}, z_{0}\right)$ obeys the outgoing wave boundary condition

$$
\Psi\left(\mathbf{r}, z_{0}\right) \sim b\left(z_{0}\right) e^{i N \mathbf{R}}, \quad R \rightarrow \infty .
$$

$\mathbf{r}$ stands for all the coordinates collectively, $\mathbf{R}$ is the radial coordinate for the outgoing electron, the (complex) coefficient $b\left(z_{0}\right)$ is proportional to the outgoing flux, and the (complex) constant $N$ acquires its explicit form depending on the potential (e.g., Coulomb, short-range, linear, etc.) $[47,54]$.

By considering either the physics of a decaying state $[52,53]$ or the reduction from Fano's stationary state formalism [47,54], two results relevant to the MEMP approach pertain to $\Psi\left(\mathbf{r} ; z_{0}\right)$. The first is that the coordinate transformation $r \rightarrow \rho=r e^{i \theta}$, first proposed for short-range potentials by Dykhne and Chaplik [55], regularizes the resonance function and makes it $\mathcal{L}^{2}$. This change in the asymptotic boundary conditions allows the direct and unique connection between $\Psi_{0}(\mathbf{r})$, the $\mathcal{L}^{2}$ wave function of the "bare" state with energy $E_{0}=\left\langle\Psi_{0}\left|H_{\text {atom }}\right| \Psi_{0}\right\rangle$ on the real axis, and $\Psi(\rho)$, the $\mathcal{L}^{2}$ resonance function with complex energy $z_{0}=\left\langle\Psi\left|H_{\text {atom }}\right| \Psi\right\rangle$ on the second Riemann sheet, with $\operatorname{Re} z_{0} \approx E_{0}$.

The second result pertains to the form of $\Psi(\boldsymbol{\rho})$. This form consists of two parts. One represents the initial state $\Psi_{0}$ before the effective interaction $V_{\text {eff }}=H-Q H Q ; Q$ $=\left|\Psi_{0}\right\rangle\left\langle\Psi_{0}\right|$, causes its decay. The other parts $X_{\text {as }}$, which is essentially a small but crucial addendum, represents the contribution from the outgoing wave. This idea was expressed in Ref. [52] as a normalization procedure subsequently named "exterior complex scaling," and in Refs. $[53,54]$ in terms of forms such as (Ref. [53], Sec. 7)

$$
\Psi=\Psi_{0}+\sum_{n} \alpha_{n} u_{n}
$$

where $u_{n}$ are trial complex basis functions. In fact, when actually constructing the Hamiltonian matrix, invariance properties such as $\left\langle\Psi_{0}(\rho)|H(\rho)| \Psi_{0}(\rho)\right\rangle$ $=\left\langle\Psi_{0}(r)|H(r)| \Psi_{0}(r)\right\rangle=E_{0}$ allow the back rotation $\rho$ $\rightarrow \rho^{*}=r e^{-i \theta}$, so that only one set of orbitals in $u_{n}$, representing the outgoing "Gamow" orbital, has its coordinates rotated by $e^{-i \theta}$. The optimization of $\Psi$ of Eq. (5) is thus done in two steps.

The first step involves the calculation of $\Psi_{0}$ of Eq. (5) with functions of real coordinates with a real energy $E_{0}$, as was emphasized in the 1978 papers [52,53]. This is a manyelectron problem with particularities, since it involves excited electronic structures representing autoionizing states. For a two- or three-electron atom, one can in principle tackle it by direct diagonalization using very large $\mathcal{L}^{2}$ basis sets and selection criteria for the relevant roots. However, it is more reliable, efficient, and general to apply state-specific methods suitable for arbitrary excited states of $\mathrm{N}$-electron atoms (see Ref. [56], and references therein). The second step involves the construction of the complex Hamiltonian matrix using Eq. (5) and its repeated diagonalization by varying $\theta$ and/or nonlinear parameters in $u_{n}$ for each expansion length, until an acceptable stability for the complex energy $z_{0}$ is found, subject to conditions

$$
\operatorname{Re} z_{0} \approx E_{0} \quad \text { and } \quad\left|\left\langle\Psi \mid \Psi_{0}\right\rangle\right|=\max .
$$

The above concepts and procedures have been adapted directly to the FII-MEMP problem by us, using $H_{\mathrm{dc}}$ and $H_{\mathrm{ac}}$ $[16-20,24,47]$ as well as the quadratic Zeeman operator [25]. Now, however, the construction and solution of the relevant matrix equations is much more demanding in practice. As regards the size of the calculation, the nature of the electromagnetic perturbing operators forces a consideration and calculation of many more state-specific $N$-electron "real" and "complex" functions. For example, in the case of the ac field, the dipole approximation requires the inclusion in a sequential manner of "Floquet blocks" of increasing angular momentum symmetry, until convergence is achieved for a given $\omega$ and $F_{\text {ac }}$.

The methodology of the MEMPT is as follows: upon perturbation by the external field, the initial bare state $\Psi_{0}$ acquires the form

$$
\begin{aligned}
\Psi_{0}(r) \rightarrow \Psi(\rho)= & \sum_{i, n} \alpha_{i, n}(\theta)\left|\Psi_{i}(\rho) ; n\right\rangle \\
& +\sum_{j, n} b_{j, n}(\theta)\left|X_{j}(\rho) ; n\right\rangle,
\end{aligned}
$$

where $\Psi_{i}$ denotes bound states (including the initial one) and localized parts of autoionizing states, $X_{j}$ denotes the $\mathcal{L}^{2}$ "scattering" states of one or two electrons in the continuum, and $n$ denotes the photon states. According to the discussion above, the $\mathcal{L}^{2} \Psi(\rho)$ satisfies the CESE

$$
\left(H_{\mathrm{ac}}(\rho)-z_{0}\right) \Psi(\rho)=0,
$$

where, formally, all complex coordinates are rotated by an angle $\theta$ sufficient to make $\Psi(\rho)$ square integrable. Substitution of expansion (7) into Eq. (8) yields the matrix complex eigenvalue MEMP equation

$$
\hat{H}_{s} \mathbf{X}=z_{0} \mathbf{X},
$$


where

$$
\hat{H}_{s}=\left[\begin{array}{ccccc}
\hat{A}+2 \omega \hat{I} & \hat{U} & & & \\
\hat{U} & \hat{A}+\omega \hat{I} & \hat{U} & & 0 \\
& \hat{U} & \hat{A} & \hat{U} & \\
0 & & \hat{U} & \hat{A}-\omega \hat{I} & \hat{U} \\
& & & \hat{U} & \hat{A}-2 \omega \hat{I}
\end{array}\right]
$$

$\hat{A}$ is the free atom Hamiltonian matrix in terms of the basis set of Eq. (5) and $\hat{U}$ is the interaction Hamiltonian matrix. $z_{0}$ is given by

$$
z_{0}=E_{0}+\Delta\left(\omega, F_{\mathrm{ac}}\right)-\frac{1}{2} i \Gamma\left(\omega, F_{\mathrm{ac}}\right),
$$

where $\Delta$ is the energy shift from $E_{0}$, and $\Gamma$ is the energy width of the dressed (resonance) state.

When using expansions such as Eq. (5) or (7) in the corresponding CESE's, it was argued in Refs. [53] and [16-19] that matrix elements involving bound-state wavefunctions remain invariant under the back-rotation $\rho e^{-i \theta}=r$, with obvious favorable consequences as to the type and economy of the computation of $z_{0}$ in the complex energy plane. In the case of the present MEMPT, the bound matrix elements have the invariance properties

$$
\begin{gathered}
\left\langle\Psi_{i}(\rho) ; n\left|H_{A}(\rho)+\hbar \omega \alpha_{\omega}^{\dagger} \alpha_{\omega}\right| \Psi_{j}(\rho) ; n^{\prime}\right\rangle \\
=\left\langle\Psi_{i}(r) ; n\left|H_{A}(\mathbf{r})+\hbar \omega \alpha_{\omega}^{\dagger} \alpha_{\omega}\right| \Psi_{j}(\mathbf{r}) ; n^{\prime}\right\rangle \\
=\left(n \hbar \omega+E_{i} \delta_{i j}\right) \delta_{n n}, \\
\left\langle\Psi_{i}(\rho) ; n\left|-\frac{1}{2} F_{a c} z e^{i \theta}\left(\alpha_{\omega}^{\dagger}+\alpha_{\omega}\right)\right| \Psi_{j}(\rho) ; n \pm 1\right\rangle \\
=\left\langle\Psi_{i}(\mathbf{r})\left|-\frac{1}{2} F_{\mathrm{ac}} z\right| \Psi_{j}(\mathbf{r})\right\rangle .
\end{gathered}
$$

Equations (11) imply that these matrix elements, including the ones involving $\Psi_{0}$, are computed only once, on the real axis, and are kept fixed when searching for $z_{0}$.

The $\mathcal{L}^{2}$ scattering states $X_{j}(\rho)$ can be written as a symmetry adapted product of a bound correlated core rotated in the complex plane and of orbitals (as yet unspecified) corresponding to each decay channel

$$
X_{j}(\rho)=X_{j}^{\text {core }}(\rho) \otimes \varepsilon l(\theta) .
$$

The $\mathcal{L}^{2}$ rotated orbital $\epsilon l(\theta)$ is expanded in terms of a basis set with real coordinates, $\varphi_{k}(r)$ :

$$
\varepsilon l(\theta)=\sum_{k} c_{k}(\theta) \varphi_{k}(r)
$$

In our work, $\varphi_{k}(r)$ are chosen as Slater orbitals and their number is augmented until stable results for the complex energy are obtained. The matrix elements involving $X_{j}(\rho)$ satisfy

$$
\begin{gathered}
\left\langle X_{i}(\rho) ; n|H(\rho)| X_{j}(\rho) ; n\right\rangle \\
=n \hbar \omega \delta_{i j}+\left\langle X_{i}^{\mathrm{core}}(r)\right. \\
\otimes \sum_{k} c_{k}^{(j)} \varphi_{k}\left(r e^{-i \theta}\right)\left|H_{A}(r)\right| X_{j}^{\mathrm{core}}(r) \\
\left.\otimes \sum_{m} c_{m}^{(j)} \varphi_{m}\left(r e^{-i \theta}\right)\right\rangle, \\
\left\langle X_{i}(\rho) ; n|H(\rho)| X_{j}(\rho) ; n \pm 1\right\rangle \\
=\left\langle X_{i}^{\text {core }}(r) \otimes \sum_{k} c_{k}^{(i)} \varphi_{k}\left(r e^{-i \theta}\right)\left|-\frac{1}{2} F_{\mathrm{ac} z}\right| X_{j}^{\mathrm{core}}(r)\right. \\
\left.\otimes \sum_{m} c_{m}^{(j)} \varphi_{m}\left(r e^{-i \theta}\right)\right\rangle, \\
\left\langle\Psi_{i}(\rho) ; n|H(\rho)| X_{j}(\rho) ; n \pm 1\right\rangle \\
=\left\langle\Psi_{i}(r)\left|-\frac{1}{2} F_{\mathrm{ac} z}\right| X_{j}^{\mathrm{core}}(r) \otimes \sum_{k} c_{k}^{(j)} \varphi_{k}\left(r e^{-i \theta}\right)\right\rangle .
\end{gathered}
$$

Expansion (7) and the above invariance properties of matrix elements reduce the MEMP problem into two overall types of computation. The first type focuses on the accurate calculation of state-specific, correlated functions for the bound and autoionizing states, thereby accounting for the bulk of the many-electron calculations which are carried out only once on the real coordinate axis. The second type aims at the optimization of the orbitals $\varphi_{k}$ and their coefficients $c_{k}^{(j)}$ which correspond to each decay channel defined by each $X_{j}^{\text {core }}$ and photon occupation number $n$. The matrix elements of the building blocks of $\hat{H}_{s}$ [Eqs. (9a, 9b)] $\hat{A}$ and $\hat{U}$ are calculated in terms of two categories of functions. The first category involves functions of real coordinates, obtained either numerically or analytically, which represent bound $\left(\Psi_{i}(r)\right)$ ionic core $\left(X_{i}^{\text {core }}(r)\right)$ or autoionizing states calculated at the multiconfigurational Hartree-Fock (MCHF) level with or without the addition of more correlation vectors. The second category contains $\left(\varphi_{k}(r)\right)$, which are rotated $\left(\varphi_{k}\left(r e^{-i \theta}\right)\right)$ while remaining $\mathcal{L}^{2}$. Because of the separation of the function spaces and of the consequences of the invariance properties, the size of final matrices in the overall calculation is sufficiently small so as to allow rigorous and reliable calculations on polyelectronic systems.

The $\theta$ dependence in $\varphi_{k}$ results in a $\theta$-dependent MEMP matrix $\hat{H}_{s}$. On the other hand, the exact eigenvalue $z_{0}$ [Eq. (10)] is $\theta$ independent. This implies that good convergence exists when for a reasonably large range of $\theta$ values, for each digonalization the complex eigenvalue corresponding to the initial bound state wave function $\Psi_{0}$ remains essentially the same. The state-specific, physically transparent choice of the function spaces of Eq. (7) allows the direct identification of the solution vector from conditions (6). 


\section{Solution of the matrix eigenvalue equation}

The infinite MEMP matrix of Eq. (9), $\hat{H}_{s}(\theta)$, should be truncated at a point which allows its practical diagonalization without sacrificing the requirement that the results have reached convergence. It follows that some thought has to be given to the way the diagonalization of $H_{s}(\theta)$ is carried out, especially since its direct diagonalization is not convenient. This inconvenience is circumvented by writing the eigenvalue equation (9) as an infinite ladder of equations

$\hat{U} \mathbf{X}_{n-1}(\theta)+(\hat{A}+n \omega \hat{I}) \mathbf{X}_{n}(\theta)+\hat{U} \mathbf{X}_{n+1}(\theta)=z_{0} \mathbf{X}_{n}(\theta)$,

where $n$ runs from $-\infty$ to $+\infty, \hat{I}$ is the unit matrix, $\mathbf{X}_{n}(\theta)$ is the part of the complexified eigenvector corresponding to photon number $n$, and $z_{0}$ is the eigenvalue. The introduction of the "shift" operators $\mathbf{P}_{n}$ and $\mathbf{Q}_{n}$ that have the property

$$
\mathbf{X}_{n}=\mathbf{P}_{n} \mathbf{X}_{n+1}, \quad \mathbf{X}_{n}=\mathbf{Q}_{n} \mathbf{X}_{n-1}
$$

results, via Eq. (15), in the recurrence relations

$$
\begin{gathered}
\mathbf{P}_{n}=-\left[\left(\hat{A}+n \omega \hat{I}-z_{0} \hat{I}+\hat{U} \mathbf{P}_{n-1}\right)^{-1}\right] \hat{U}, \\
\mathbf{Q}_{n}=-\left[\left(\hat{A}+n \omega \hat{I}-z_{0} \hat{I}+\hat{U} \mathbf{Q}_{n+1}\right)^{-1}\right] \hat{U} .
\end{gathered}
$$

Thus, the diagonalization of the infinite matrix $\hat{H}_{s}$ is reduced to first solving an eigenvalue equation, of finite dimension [Eq. (15), $n=0$ ]

$$
\hat{U} \mathbf{X}_{-1}+\hat{A} \mathbf{X}_{0}+\hat{U} \mathbf{X}_{1}=z_{0} \mathbf{X}_{0},
$$

which is then transformed, via the "shift" operators $\mathbf{P}_{n}$ and $\mathbf{Q}_{n}$, to

$$
\left(\hat{U} \mathbf{P}_{-1}+\hat{A}+\hat{U} \mathbf{Q}_{1}\right) \mathbf{X}_{0}=z_{0} \mathbf{X}_{0} .
$$

The matrices $\mathbf{P}_{-1}$ and $\mathbf{Q}_{1}$ are infinite continued fractions, as can be seen from Eq. (17), with $n$ ranging from $-\infty$ to $+\infty$. The inverse of the matrix,

$$
\mathbf{M}_{0}=z_{0} \hat{I}-\hat{A}-\hat{U} \mathbf{P}_{-1}-\hat{U} \mathbf{Q}_{1},
$$

is the "elastic resolvent" of the continued-fraction perturbation theory in matrix form [3]. For the model case of $2 \times 2$ matrices, we can solve analytically Eqs. (18b) and (18c) by setting the determinant of $\mathbf{M}_{0}$ equal to zero. The results thus obtained are identical to those of Autler and Townes [57].

The solution of the eigenvalue equation (18b) must be obtained self-consistently, since the matrices $\mathbf{P}_{-1}$ and $\mathbf{Q}_{1}$ depend on the eigenvalue $z_{0}$. In practice, the infinite continued fractions $\mathbf{P}_{-1}$ and $\mathbf{Q}_{1}$ are truncated at some large value of $N$, for which we assume that

$$
\mathbf{P}_{-|N|-1}=\mathbf{Q}_{|N|+1}=\mathbf{0} .
$$

The calculation is checked so as to ensure that indeed (18d) is satisfied after some large value of $N$.
We emphasize that this method requires the invertion and diagonalization of matrices of the order of $\hat{A}$ and $\hat{U}$, while taking into account the photon states to any order. Thus, the computational efforts in the MEMPT focus on the accurate construction of the block matrices $\hat{A}$ and $\hat{U}$, which is achieved by incorporating all those correlated wave functions which affect the multiphoton process.

\section{Calculation of linear and nonlinear polarizabilities}

The calculated complex eigenvalue of Eq. (10) provides the multiphoton ionization rates, $\Gamma\left(\omega, F_{a c}\right)$, as well as the energy shift $\Delta\left(\omega, F_{a c}\right)$. For the static case, the expansion in Taylor series of $\Delta_{\text {static }}$ provides the definition of the linear and nonlinear polarizabilities [see, e.g., Ref. [58]]. For atoms,

$$
\Delta_{\text {static }}=-(1 / 2 !) \alpha F_{0}^{2}-(1 / 4 !) \gamma F_{0}^{4}-(1 / 6 !) \delta F_{0}^{6}-\cdots .
$$

For the dynamic case, the solution of Eq. (9) can be achieved with more than one ac field of different frequencies and/or static fields. Until now, we have restricted our work to a maximum of one ac field and one dc field. When one ac field of frequency $\omega$ is present, we have used the following series expansion for $\Delta(\omega)[19,20]$ :

$$
\begin{aligned}
\Delta(\omega)= & -(1 / 2 !) \alpha(\omega)\left(\overline{F_{0} \cos (\omega t)}\right)^{2} \\
& -(1 / 4 !) \gamma(\omega)\left(\overline{F_{0} \cos (\omega t)}\right)^{4}-\cdots \\
= & -(1 / 2 !) \alpha(\omega)(1 / 2) F_{0}^{2}-(1 / 4 !) \gamma(\omega)(3 / 8) F_{0}^{4} \\
& -(1 / 6 !) \delta(\omega)(5 / 16) F_{0}^{6}-\cdots,
\end{aligned}
$$

where in going from Eq. (20a) to Eq. (20b) we have averaged over an optical cycle. The functions $\alpha(\omega)$ and $\gamma(\omega)$ represent the first two expansion coefficients of induced polarization for linearly polarized light averaged over an optical cycle. We point out that in the MEMPT, the values of $\omega$ can go beyond the ionization threshold, $E_{t h r}$, without any difficulty, as indeed was demonstrated with $\mathrm{Li}^{-}[19]$ and $\mathrm{H}^{-}$ [20]. This is because the contribution of the continuous spectrum is achieved rigorously, without any changes in the analytic structure of frequency-dependent quantities. Conversely, calculations of frequency-dependent linear and nonlinear polarizabilities via conventional perturbation theory and Green's-function expansions must deal reliably with the problem of divergences in matrix elements involving scattering states.

For approaches based on perturbation theory, the linear and nonlinear coefficients of the series expansion in the field are obtained individually from expressions corresponding to different observable processes. Specifically, the hyperpolarizability tensor $\gamma_{\alpha \beta \gamma \delta}\left(-\omega_{\sigma}, \omega_{1}, \omega_{2}, \omega_{3}\right)$, is defined $[1,2,14]$ by the equation

$$
\begin{aligned}
P_{\alpha}\left(\omega_{\sigma}\right)= & K \gamma_{\alpha \beta \gamma \delta}\left(-\omega_{\sigma} ; \omega_{1}, \omega_{2}, \omega_{3}\right) \\
& \times E_{\beta}\left(\omega_{1}\right) E_{\gamma}\left(\omega_{2}\right) E_{\delta}\left(\omega_{3}\right),
\end{aligned}
$$


where $\alpha, \beta, \gamma$, and $\delta$ are atom- or laboratory-fixed axes $(\mathrm{x}, \mathrm{y}, \mathrm{z}), E_{\beta}\left(\omega_{1}\right)$ is the $\beta$ component of the applied field with frequency $\omega_{1}, P_{\alpha}\left(\omega_{\sigma}\right)$ is the induced polarization with frequency $\omega_{\sigma}\left[\omega_{\sigma}=\omega_{1}+\omega_{2}+\omega_{3}\right]$, and $K$ is a numerical factor. Depending on the combination of photon frequencies and of transition processes, $\gamma$ expresses the dynamic polarization for different phenomena. For example, the dc Kerr effect is represented by $\gamma(-\omega ; \omega, 0,0)$, the degenerate four-wave mixing (DFWM) by $\gamma(-\omega ;-\omega, \omega, \omega)$, the electric-field-induced second-harmonic generation by $\gamma(-2 \omega ; 0, \omega, \omega)$ and the thirdharmonic generation (THG) by $\gamma(-3 \omega ; \omega, \omega, \omega)$.

The nonperturbative MEMPT incorporates field-induced effects to all orders. The calculation directly yields $\Delta$ and $\Delta(\omega)$, and the quantities $\alpha, \alpha(\omega)$, and $\gamma, \gamma(\omega)$ may then be obtained, for small field strength values, by fitting the energy shifts to a polynomial of high degree. For linearly polarized monochromatic light, the hyperpolarizability tensor component thus obtained is $\gamma_{z z z z}$, and represents a frequencydependent superposition of the effects of DFWM and THG, for which $\left|\omega_{1}\right|=\left|\omega_{2}\right|=\left|\omega_{3}\right|=\omega$. Therefore, a direct comparison with the results of LOPT, (e.g., Ref. [14]), where hyperpolarizabilities for these processes are calculated separately, cannot be made. However, for certain values of frequencies, it happens that only one process dominates, (e.g., when the denominator of the corresponding perturbation theory formulas become very small), in which case $\gamma(\omega)$ represents essentially only this process (see next section).

\section{RESULTS}

According to the description of Sec. II, MEMPT is implemented ab initio by choosing, manipulating, and optimizing suitable one-electron and $\mathrm{N}$-electron basis sets. Calculations such as the ones carried out in this work are time consuming not only because of the need to account for the electronic structure and electron correlation of many states, but also because of the number of properties examined over large ranges of values for $\omega$ and $I$. Therefore, in order to make such a project worth pursuing, it is crucial to use function spaces which, on the one hand, render the calculations tractable, and, on the other hand secure that the results contain quantitatively all the significant physical information. As an indicative test of the level of accuracy of these calculations, we chose the comparison between the results of Bishop and Lam [14(a)] and our result for the dynamic polarizability, $\alpha(\omega)$. They used LOPT with large expansions of carefully optimized $r_{12}$-correlated functions obtained earlier by Thakkar and Smith. Table II shows that the average deviation is only $0.35 \%$, with our numbers being systematically smaller except for $\omega=0.75$ a.u. (see below). The function space used here consisted of the following two-electron functions.

(1) The initial state $\Psi_{0} H^{1} S$ was represented by a correlated ten-term numerical MCHF wave function [59] with the configurations $1 s^{2}, 2 s^{2}, 2 p^{2}, 3 s^{2}, 3 p^{2}, 3 d^{2}, 4 s^{2}, 4 p^{2}$, $4 d^{2}$, and $4 f^{2}$. This function contains essentially all the radial and angular correlations which play a role when the bare ground state is perturbed by the external field.

(2) $\Psi_{i}$ of Eq. (7) consist of singly excited Rydberg con-

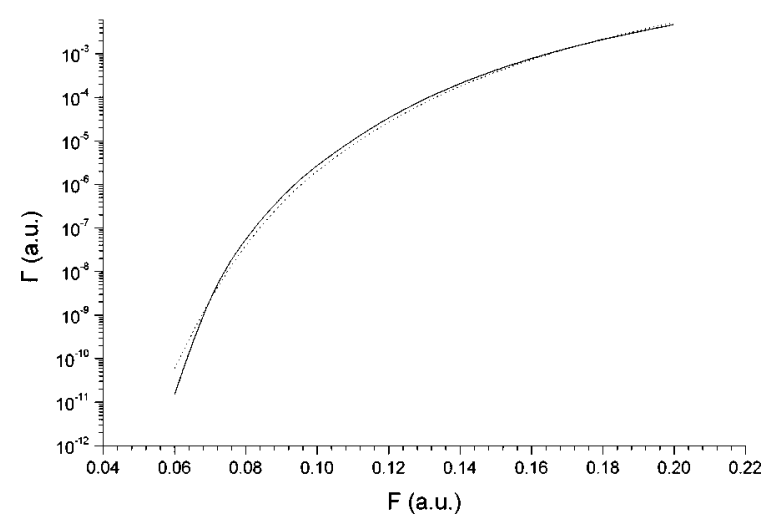

FIG. 1. He tunneling rates ( $\Gamma$ in a.u.) as a function of the dcfield strengths ( $F$ in a.u.). The dotted line represents the rates which were obtained from the Ammosov-Delone-Krainov semiclassical formula [61]. The solid line represents the rates which were obtained from the present MEMPT.

figurations and of doubly excited configurations representing autoionizing states and correlations. In the He Rydberg states, electron correlation is not significant for the properties under consideration. What is important is to have good radials for the Rydberg electron. This is achieved here via the use of numerical Hartree-Fock functions $1 s n l{ }^{1} L$ with $n=2,3, \ldots, 6$ and $L=l=0, \ldots, 5$. The remainder of the function space, which is made to mix via $H_{\mathrm{ac}}$ is represented by 82 excited configurations $\mathrm{v}_{l} \mathrm{v}_{l}^{\prime 1} L$, where $\mathrm{v}_{l}$ are virtual orbitals, with $l=0, \ldots, 3$, and $L=0, \ldots, 4$.

(3) $X_{j}$ of Eq. (7) are represented by configurations of the type $1 s \otimes \varepsilon l$, where the orbital $\varepsilon l$ is expanded in terms of Slater-type orbitals (STO's)

$$
\varphi_{k}\left(\rho^{*}\right)=\varphi_{k}\left(r e^{-i \theta}\right)=c_{k}(\theta) r^{k} e^{-\alpha \rho^{*}} .
$$

For each $l(l=0, \ldots, 5)$ the continuum orbitals were represented by ten $\varphi_{k}\left(\rho^{*}\right)$, except for $l=5$, for which eight $\varphi_{k}$ were used.

The convergence of the results was tested with respect to the rotation angle $\theta$ and the STO exponent $\alpha$, and with respect to the number $N$ of the blocks of the infinite matrix used [see Eqs. (9b) and (18d)]. The convergence is achieved when the results are independent of $\theta, \alpha$, and $N$ over a reasonable range of parameter values. For most of the results, this was accomplished for $|N| \geqslant 10, \alpha$ around 1.5 and $\theta$ around 0.3 rads.

\section{A. dc-field tunneling rates}

The only earlier quantum-mechanical work for this property on He is that of Ref. [60], where the same method was used but with a smaller basis set and a less extensive optimization search in parameter space necessitated at the time by restricted computer power. In Fig. 1 we compare the present $a b$ initio tunneling rates for He with semiclassical results calculated here according to the widely used Ammosov-Delone-Krainov (ADK) tunneling rate formula [61]. The validity of the ADK formula in the case of $\mathrm{He}$ is thus tested for the range of field strengths used here. The 


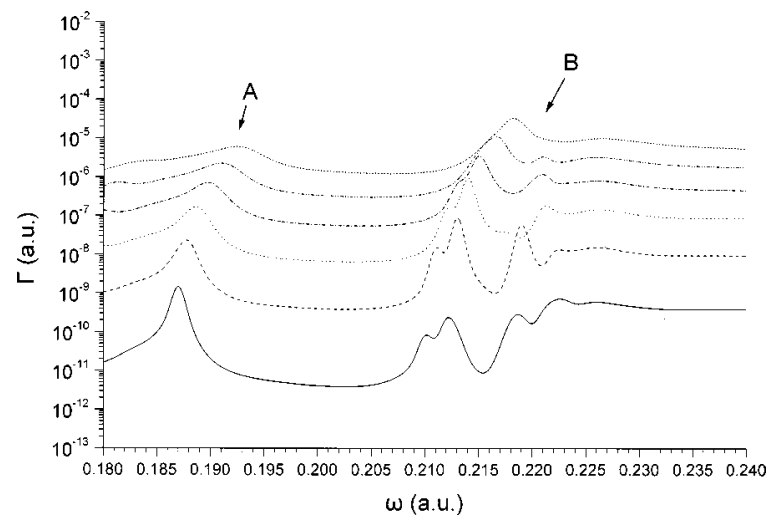

FIG. 2. He MPI rates ( $\Gamma$ in a.u.) in the laser frequency ( $\omega$ in a.u.) region, where at least five photons are necessary for ionization. The different curves correspond to different laser intensities $I$. Specifically, the solid line curve was obtained for $I=1.4 \times 10^{13} \mathrm{~W} / \mathrm{cm}^{2}$, the dashed line for $I=3.15 \times 10^{13} \mathrm{~W} / \mathrm{cm}^{2}$, the dotted line for $I=5.6$ $\times 10^{13} \mathrm{~W} / \mathrm{cm}^{2}$, the dash-dotted line for $I=8.75 \times 10^{13} \mathrm{~W} / \mathrm{cm}^{2}$, the dash-dotted-dotted line for $I=1.26 \times 10^{14} \mathrm{~W} / \mathrm{cm}^{2}$, and the short dashed line for $I=1.715 \times 10^{14} \mathrm{~W} / \mathrm{cm}^{2}$. The peaks denoted by $A$ correspond to $(4+1)$ REMPI with the $1 s 2 s{ }^{1} S$ state. Similarly, the peaks denoted by $B$ correspond to (4+1) REMPI with $1 s 3 s{ }^{1} S$, $1 s 3 d{ }^{1} D, 1 s 4 s^{1} S$, and $1 s 4 d{ }^{1} D$ states.

agreement between the ab initio MEMPT results and the semiclassical ones is surprisingly good. Our calculations were systematic and detailed in the variation of parameters with special attention to the weak-field regime, where the imaginary part of the complex eigenvalue becomes very small. It is in the weak-field regime that the present results correct the ones reported in Ref. [60].

\section{B. ac-field multiphoton ionization rates}

The large ranges of frequencies $[\omega=248 \mathrm{~nm}(\sim 5 \mathrm{eV})$ to $40 \mathrm{~nm}(\sim 26.4 \mathrm{eV})]$ and of intensities $\left(I=3.5 \times 10^{12}-1.715\right.$ $\times 10^{14} \mathrm{~W} / \mathrm{cm}^{2}$ ) which were used in the MEMPT calculations of MPI rates were chosen so as to mark out different types of features which are characteristic of the high-order, nonlinear nature of the response. The results are shown in Figs. 2-5. Each one corresponds to a particular region of $\omega$ values. To our knowledge, such predictions are reported here for the first time.

Figure 2 shows the rates, for peak intensities in the range $1.4 \times 10^{13}-1.715 \times 10^{14} \mathrm{~W} / \mathrm{cm}^{2}$, in the frequency region where at least five photons are necessary for ionization. Especially for low intensities, a series of identifiable peaks emerge from a more or less smooth background. This is a manifestation of a 4+1 resonance-enhanced multiphoton ionization (REMPI) where the field energy of four photons comes to resonance with the $1 s 2 s{ }^{1} S, 1 s 3 s^{1} S, 1 s 3 d{ }^{1} D$, $1 s 4 s{ }^{1} S$, and $1 s 4 d{ }^{1} D$ states, while the fifth photon drives the He atom to the continuum. As the intensities increase, the positions of these peaks shift to higher energies, and the discrete structures become smoother and broader. Finally, for $1.715 \times 10^{14} \mathrm{~W} / \mathrm{cm}^{2}$, the rate function is smooth, with only two broad bumps; one representing the $1 s 2{ }^{1} S$ intermediate state and the other representing the collective contribution

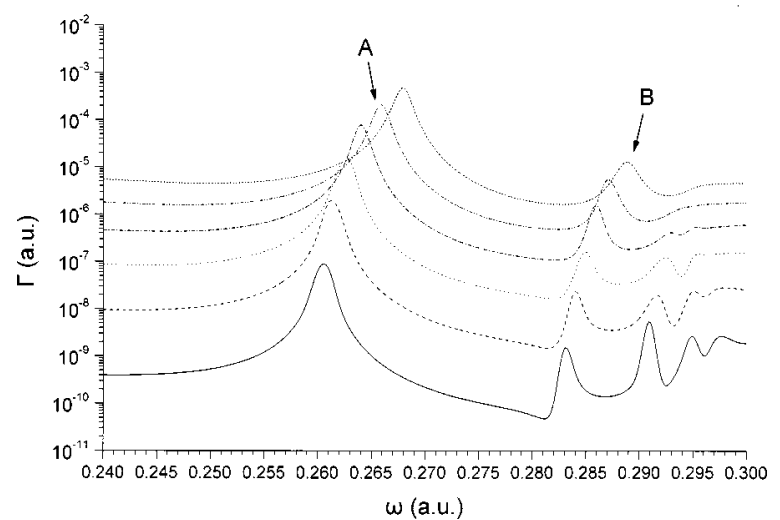

FIG. 3. As in Fig. 2 but in the frequency region where at least four photons are necessary for ionization. The peaks denoted by $A$ correspond to $(3+1)$ REMPI with the $1 s 2 p{ }^{1} P^{o}$ state. Similarly, the peaks denoted by $B$ correspond to $(3+1)$ REMPI with $1 s 3 p^{1} P^{o}, 1 s 4 p{ }^{1} P^{o}, 1 s 4 f^{1} F^{o}, 1 s 5 p{ }^{1} P^{o}$, and $1 s 5 f{ }^{1} F^{o}$ states.

from the ${ }^{1} S$ and ${ }^{1} D$ Rydberg states for $n=3$ and 4 . The overall behavior of $\Gamma(\omega)$ is thus in accordance with the expected upward shift, and the with the width broadening that the Rydberg states undergo as intensity increases.

In Fig. 3, the frequencies cover the energy necessary for at least four-photon ionization. The intensities are in the range of $1.4 \times 10^{13}-1.715 \times 10^{14} \mathrm{~W} / \mathrm{cm}^{2}$. The $(3+1)$-photon REMPI with intermediate state the $1 s 2 p{ }^{1} P^{o}$ is reflected in the first peak. As the intensity increases, the position of this peak shifts to higher energies, but its width broadens relatively little. The second peak corresponds to the $(3+1)$ photon REMPI with intermediate states the $1 s 3 p{ }^{1} P^{o}$, $1 s 4 f^{1} F^{o}, 1 s 4 p^{1} P^{o}, 1 s 5 f^{1} F^{o}$, and $1 s 5 p^{1} P^{o}$. The near degeneracy of the states belonging to the fourth and higher

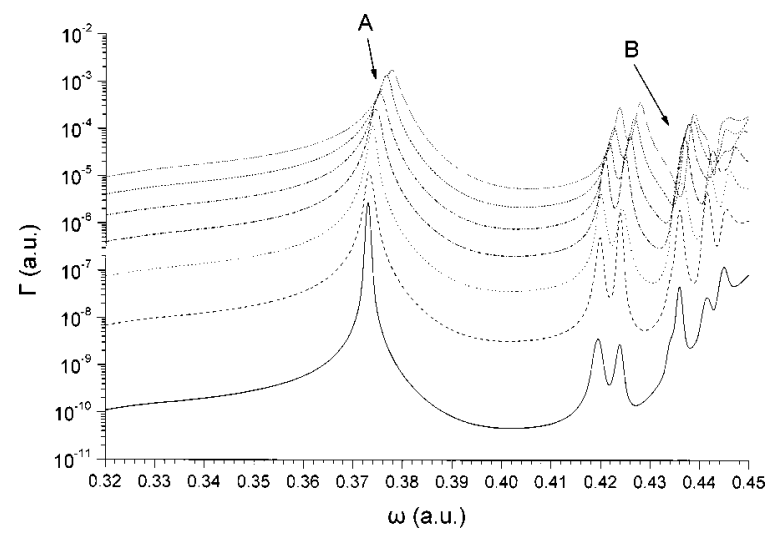

FIG. 4 . He MPI rates ( $\Gamma$ in a.u.) in the laser frequency ( $\omega$ in a.u.) region where at least three photons are necessary for ionization. The solid line curve was obtained for peak intensity $I=3.5$ $\times 10^{12} \mathrm{~W} / \mathrm{cm}^{2}$, the dashed line for $I=1.4 \times 10^{13} \mathrm{~W} / \mathrm{cm}^{2}$, the dotted line for $I=3.15 \times 10^{13} \mathrm{~W} / \mathrm{cm}^{2}$, the dash-dotted line for $I=5.6$ $\times 10^{13} \mathrm{~W} / \mathrm{cm}^{2}$, the dash-dotted-dotted line for $I=8.75 \times 10^{13} \mathrm{~W} /$ $\mathrm{cm}^{2}$, the short dashed line for $I=1.26 \times 10^{14} \mathrm{~W} / \mathrm{cm}^{2}$, and the short dotted line for $I=1.715 \times 10^{14} \mathrm{~W} / \mathrm{cm}^{2}$. The peaks denoted by $A$ correspond to $(2+1)$ REMPI with the $1 s 2 s{ }^{1} S$ state. Similarly, the peaks denoted by $B$ correspond to $(2+1)$ REMPI with $1 s n s{ }^{1} S$ and 1 snd ${ }^{1} D(n=3, \ldots, 6)$ states. 


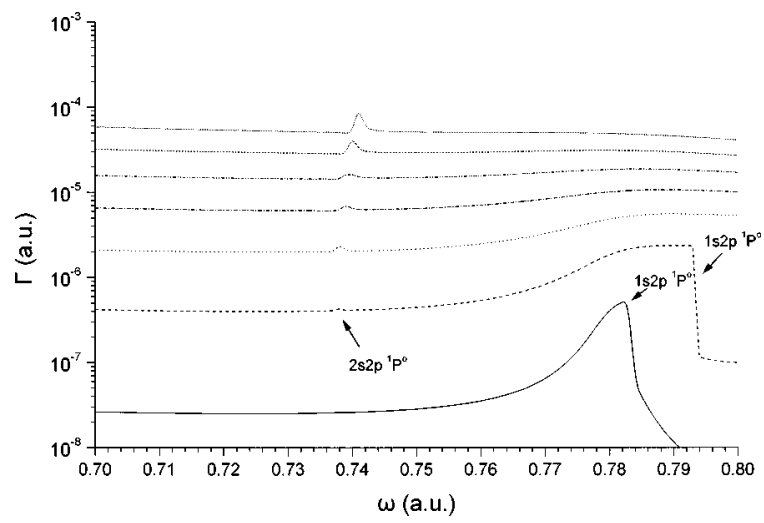

FIG. 5. As in Fig. 4 but in the frequency region where at least two photons are necessary for ionization. A peak emerges from a smooth background, just below $\omega=0.74$ a.u., as the intensity increases. It is a manifestation of a three-photon process in which the $2 s 2{ }^{1} P^{o}$ autoionizing state is coupled resonantly with the $\mathrm{He}$ ground state.

shells results in one peak for each shell. Again, the discrete structures for $n=4$ and 5 that appear for low intensities are wiped out as the intensity increases.

In Fig. 4 the frequency range corresponds to ionization with at least three photons. Results from seven intensity values between $3.5 \times 10^{12}$ and $1.715 \times 10^{14} \mathrm{~W} / \mathrm{cm}^{2}$ are shown. The same picture as in Figs. 2 and 3 appears, where the intermediate states for the 2+1 REMPI are the $1 s 2 s{ }^{1} S$, $1 s n s{ }^{1} S$, and $1 s n d{ }^{1} D n=3, \ldots, 6$ states. For $n \geqslant 4$, the peaks for the ${ }^{1} S$ and ${ }^{1} D$ states are indistinguishable. Again, the peaks shift to higher energies and broaden with increasing intensity.

Figure 5 shows $\Gamma(\omega)$ for $\omega$ values where at least two photons are required for ionization. Now, apart from the $1+1$ REMPI, which involves the appearance and disappearance (for higher intensities) of the $1 s 2 p{ }^{1} P^{o}$ discrete intermediate state, an additional structure appears at $\omega \sim 0.74$ a.u. as the field intensity increases. This structure is absent for field intensities of the order of $3.5 \times 10^{12} \mathrm{~W} / \mathrm{cm}^{2}$ or smaller, where $\Gamma(\omega)$ increases smoothly and quadratically with intensity. The interpretation of this is that it is a manifestation of a higher-order effect, whereby the autoionizing state $2 s 2 p{ }^{1} P^{o}$, whose field-free position is around $60.2 \mathrm{eV}$, is coupled with the $\mathrm{He}^{1} S$ ground state via a three-photon process. Three photons of $\omega \sim 0.74$ a.u. add to $\sim 60.4 \mathrm{eV}$. Apart from energetics and symmetry we also tested this interpretation by removing from expansion (7) the correlated function representing the $2 s 2 p{ }^{1} P^{o}$ state. In this case, the peak disappeared. This type of higher-order contribution to the ionization rate of $\mathrm{He}$ for high intensities was first reported by Purvis et al. [27], whose calculations combine CCR Floquet and $R$-matrix procedures. In their study, they observed the effect of the two-photon coupling between the ${ }^{1} S$ ground state and the lowest ${ }^{1} D$ autoionizing state in a one-photon ionization process.

We point out that this interplay between the field-free spectrum and the field characteristics of frequency and intensity is brought out rather clearly when the state-specific approach to the field-atom interaction problem is applied,

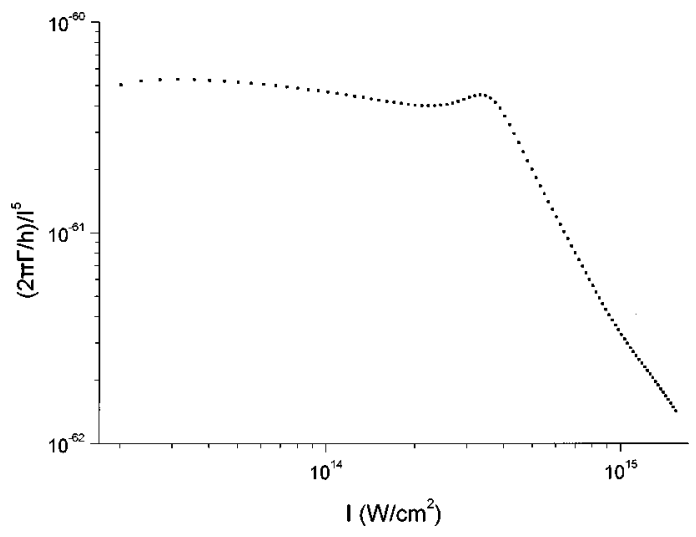

FIG. 6. The quantity $(\Gamma / \hbar) / I^{5}$ as a function of intensity $I$ for $\omega=0.2$ a.u. For this frequency at least five photons are necessary for ionization. As expected, in the low-intensity region where the $I^{5}$ power law of the five-photon ionization rate is valid, the quantity $(\Gamma / \hbar) / I^{5}$ is essentially independent of $I$.

where the fundamental ingredient is the form of Eq. (7) (see Ref. [21] and subsequent publications for the state-specific expansion approach to the solution of the time-dependent Schrödinger equation).

On the other hand, trial MEMPT calculations have indicated that difficulties with convergence and with interpretation may occur even when a faithful wave-function representation of the discrete, autoionizing, and scattering states is used as input. This is when the strength of the perturbation due to operators $F$ is such that, in combination with spectra features of the system and with the values of $\omega$, it causes strong mixing of states and large and possibly abrupt energy shifts. In such cases, the adiabatic criteria (6) must be applied with great detail (something which is computationally expensive), and must be supplemented by additional information on the solutions.

Finally, for each frequency, the laser intensity $\left(I_{\mathrm{LOPT}}\right)$ at which the $I^{k}$-power "law" of the $k$-photon ionization rate breaks down, is a parameter that determines the domain of the validity of the LOPT, except when intermediate resonances with excited states are present. For example, in Fig. 6 the quantity $(\Gamma / \hbar) / I^{5}$ is given as a function of intensity $I$ for $\omega=0.2$ a.u. Around $I=4 \times 10^{14} \mathrm{~W} / \mathrm{cm}^{2}$, the $I^{5}$ power "law", breaks down. More examples are given in Table I, where

TABLE I. The intensity $I_{\text {LOPT }}$ at which the $\Gamma \sim I^{k}$ power "law" breaks down. The values of $\omega$ were chosen so as to avoid, at least for low intensities, any Rydberg states coming into resonance.

\begin{tabular}{ccc}
\hline \hline$\omega$ (in a.u.) & $k$ & $\begin{array}{c}I_{\text {LOPT }} \\
\text { (in W/cm }\end{array}$ \\
\hline 0.184 & 5 & $1.0 \times 10^{14}$ \\
0.200 & 5 & $4.0 \times 10^{14}$ \\
0.240 & 4 & $5.0 \times 10^{14}$ \\
0.320 & 3 & $1.0 \times 10^{14}$ \\
0.400 & 3 & $3.0 \times 10^{14}$ \\
0.600 & 2 & $8.0 \times 10^{14}$ \\
0.800 & 2 & $1.0 \times 10^{14}$ \\
\hline \hline
\end{tabular}


TABLE II. Comparison of the results for the dynamic polarizability, $\alpha(\omega)$, obtained from the nonperturbative many-electron many-photon theory presented here and from the LOPT expression with $r_{12}$ correlated functions, calculated by Bishop and Lam[14(a)]. The comparison is up to $\omega=0.75$ a.u., where the LOPT calculations stopped because of the proximity to the excited state $1 s 2 p{ }^{1} P^{o}$. Our calculations actually went up to $\omega=0.97$ a.u. (see Fig. 7).

\begin{tabular}{ccl}
\hline \hline$\omega$ (a.u.) & $\begin{array}{c}\text { Bishop and } \\
\text { Lam [14(a) }\end{array}$ & \multicolumn{1}{c}{$\begin{array}{c}\text { This } \\
\text { work }\end{array}$} \\
\hline 0.20 & 1.448341 & 1.44388 \\
0.25 & 1.488335 & 1.48364 \\
0.30 & 1.540981 & 1.5360 \\
0.35 & 1.609325 & 1.60396 \\
0.40 & 1.697985 & 1.69204 \\
0.45 & 1.814214 & 1.8078 \\
0.50 & 1.970037 & 1.96236 \\
0.55 & 2.186990 & 2.17792 \\
0.60 & 2.508292 & 2.4972 \\
0.65 & 3.037345 & 3.02336 \\
0.70 & 4.111021 & 4.09692 \\
0.75 & 8.014127 & 8.19568 \\
\hline \hline
\end{tabular}

$I_{\text {LOPT }}$ means the value of $I$ up to which LOPT holds. The values of $\omega$ were chosen so as not to have any Rydberg states coming into resonance. For these frequencies, the domain of validity of the LOPT is up to $10^{14}-10^{15} \mathrm{~W} / \mathrm{cm}^{2}$. Systematic calculations concerning the peculiarities of the domain of the validity of the LOPT are under way and, hopefully, results will be reported in a future publication.

\section{Dynamic polarizabilities and hyperpolarizabilities}

Table II compares our results for $\alpha(\omega)$ with those of Bishop and Lam [14(a)] up to $\omega=0.75$ a.u. (below the ionization threshold), where the LOPT calculations of Ref. $[14(\mathrm{a})]$ stop. The agreement is very good. Figure 7 shows the MEMPT $\alpha(\omega)$ in the frequency range $0.17-0.97$ a.u. i.e., below and above the ionization threshold of $\mathrm{He}^{1} S$. The ex-

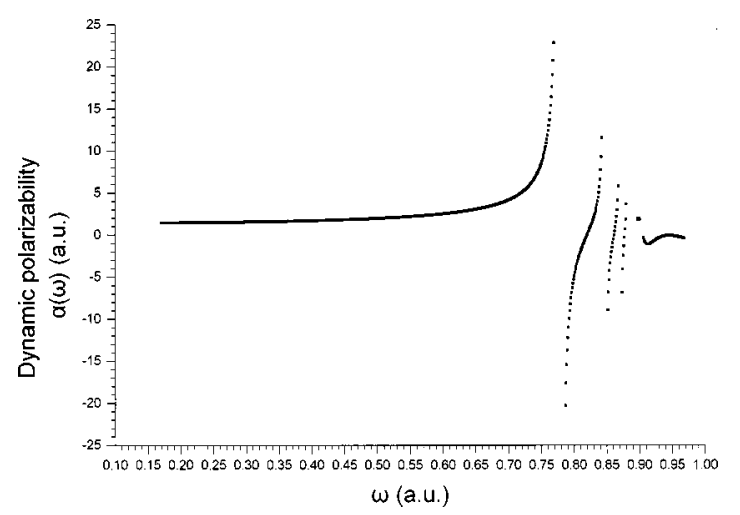

FIG. 7. He ground-state dynamic polarizability $\alpha(\omega)$, defined by Eq. (20b), below and above the ionization threshold. The strong variations which start just above $\omega=0.75$ a.u. are a manifestation of the one-photon resonant excitation of the $1 s n p{ }^{1} P^{o}$ states.

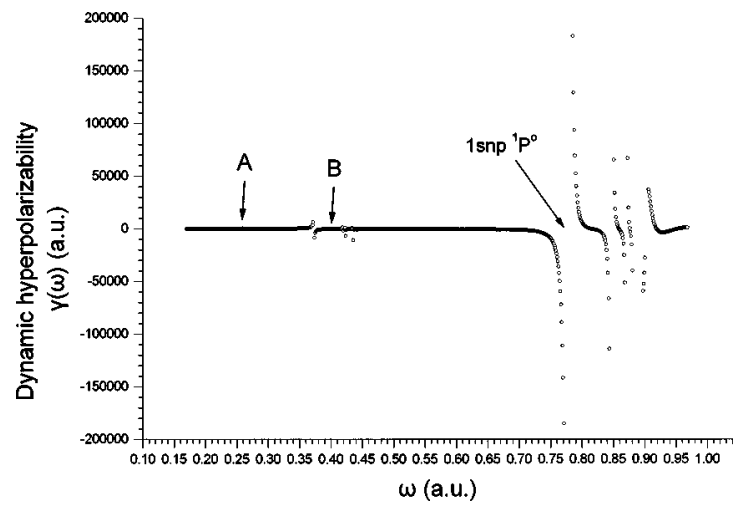

FIG. 8. He ground-state dynamic hyperpolarizability $\gamma(\omega)$, defined by Eq. (20b), below and above the ionization threshold. The variations of $\gamma(\omega)$ for $\omega>0.75$ a.u. correspond to the one-photon resonant excitation of the $1 \mathrm{snp}{ }^{1} P^{o}$ states. The structures denoted by $(A)$ and $(B)$ are shown in more detail in Figs. 9 and 10, respectively.

treme variations which start around 0.75 a.u. are the fingerprints of the one-photon resonance excitation of the 1 snp ${ }^{1} P^{o}$ states.

The calculated dynamic hyperpolarizability [Eq. (20b)], $\gamma(\omega)$, is the $z z z z$ component of the tensor, and constitutes a linear combination of the perturbation theory terms representing the processes DFWM and THG. Their presence is revealed by examining Fig. 8. The variations of $\gamma(\omega)$ for frequencies larger than 0.75 a.u. correspond to one-photon resonance excitation with the 1 snp ${ }^{1} P^{o}$ states. The structures denoted $A$ around $\omega=0.25$ a.u. are shown magnified in Fig. 9. The first, and more pronounced, structure $(\omega \sim 0.26$ a.u.) corresponds to the three-photon excitation of the $1 s 2 p{ }^{1} P^{o}$ state, and is due to the THG terms that are present in the dynamic hyperpolarizability defined by Eq. (20). Bishop and Lam [14(a)] explicitly computed the THG term up to $\omega=0.25$ a.u. However, direct comparison between their THG results and ours for $\gamma(\omega)$ is not possible.

Structures $B$ for frequency values just above $\omega=0.34$ a.u. are shown in detail in Fig. 10. These correspond to the twophoton resonance excitation of the $1 s 2 s{ }^{1} S, 1 s n s{ }^{1} S$, and 1 snd ${ }^{1} D(n=3,4$, and 5) states, and have their origin in the DFWM terms that are present in our $\gamma(\omega)$. Again, the values of DFWM computed in Ref. [14(a)] are not directly comparable with the $\gamma(\omega)$ of this calculation.

\section{SYNOPSIS}

We have presented a large amount of experimentally verifiable information for physical quantities related to the linear and nonlinear response of He to strong $\mathrm{dc}$ and ac fields. These results were obtained nonperturbatively, by applying a MEMPT which is directly applicable to ground as well as excited states of arbitrary electronic structures. We have shown how this can be done economically and generally, by systematically choosing and optimizing appropriate forms and parts of one-electron and $N$-electron functions. The key features of the theory are the following.

(i) In analogy with work on the theory and calculation of 


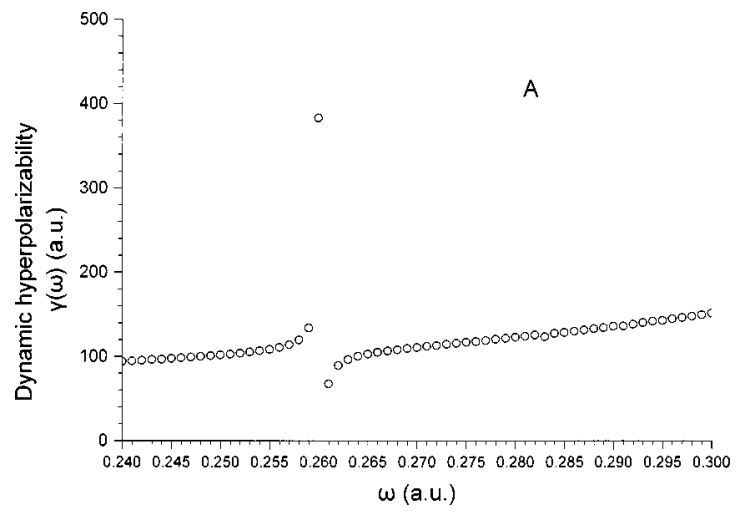

FIG. 9. Structure $A$ of the He ground-state dynamic hyperpolarizability $\gamma(\omega)$ (Fig. 8) appears in the frequency region where a resonant three-photon excitation of the $1 s 2 p{ }^{1} P^{o}$ state occurs. It is due to the THG terms that are present in the $\gamma(\omega)$ of Eq. (20b).

field-free resonance states, the problem is reduced to finding the complex eigenvalue of a state-specific matrix equation [Eq. (9)] using as trial function the expansion (7).

(ii) The $\Psi(\rho)$ of Eq. (7) consists of wave functions for the discrete, autoionizing, and free states of the system, with all the significant electronic structure and correlation effects for each case included. The theory and methods of computation of state-specific correlated wave functions based on MCHF solutions, which comprise the zero-order approximation, are described in Ref. [56] and references therein.

(iii) The solution of Eq. (9) and the overall calculation in

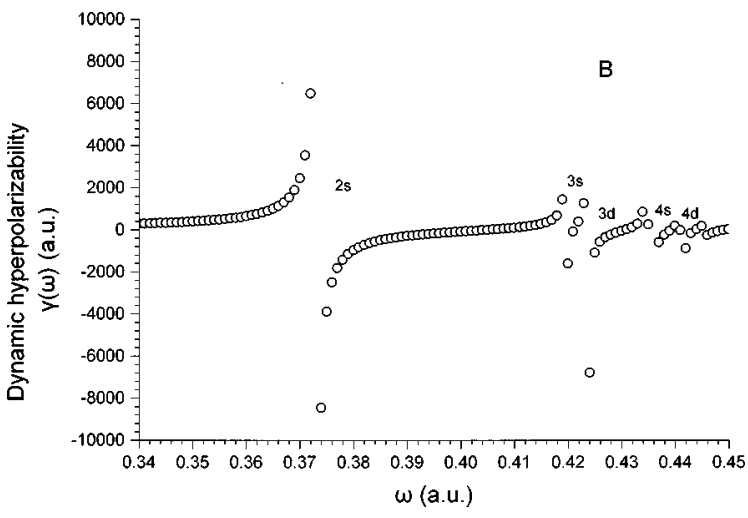

FIG. 10. Structure $B$ of the $\gamma(\omega)$ (Fig. 8) appears in the frequency region where a resonant two-photon excitation of the 1 sns ${ }^{1} S$ and 1 snd ${ }^{1} D(n=3,4$, and 5) states occurs. It is due to the DFWM terms that are present in $\gamma(\omega)$ of Eq. (20b).

the complex energy plane are simplified and made tractable and systematic due to the matrix element properties of Eqs. (11) and (14).

(iv) The combination of (i)-(iii) allows one to delineate the various contributions and to understand the interplay between electronic structure and state symmetries on the one hand, and field characteristics on the other hand.

\section{ACKNOWLEDGMENT}

One of us (S.I.T.) expresses his gratitude to the Onassis Foundation, Athens, Greece, for financial support.
[1] D. C. Hanna, M. A. Yuratich, and D. Cotter, Nonlinear Optics of Free Atoms and Molecules (Springer, Berlin, 1979).

[2] N. C. Manakov, V. D. Ovsiannikov, and L. P. Rapoport, Phys. Rep. 141, 319 (1986).

[3] F. H. Faisal, Theory of Multiphoton Processes (Plenum, New York, 1987).

[4] Atoms in Strong Fields, edited by C. A. Nicolaides, C. W. Clark, and M. H. Nayfeh (Plenum, New York, 1990).

[5] Theory of High-Order Processes in Atoms in Intense Laser Fields, edited by K. Kulander and A. L'Huillier, special issue of J. Opt. Soc. Am. B. 7 (4) (1990).

[6] Atoms in Intense Laser Fields, edited by M. Gavrila (Academic, New York, 1992).

[7] A. G. Faishtein, N. L. Manakov, V. D. Ovsiannikov, and L. P. Rapoport, Phys. Rep. 210, 111 (1992).

[8] D. M. Bishop, Adv. Quantum Chem. 25, 1 (1994).

[9] Y. Gontier, N. K. Rahman, and M. Trahin, Phys. Rev. A 34, 1112 (1986).

[10] E. Karule, J. Phys. B 21, 1997 (1988).

[11] H. J. Silverstone, in Atoms in Strong Fields (Ref. [4]), p. 295.

[12] J. N. Silverman and C. A. Nicolaides, in Atoms in Strong Fields (Ref. [4]), p. 309; Chem. Phys. Lett. 184, 321 (1991).

[13] L. Pan, K. T. Taylor, and C. W. Clark, J. Opt. Soc. Am. B 7, 509 (1990).

[14] (a) D. M. Bishop and B. Lam, Phys. Rev. A 37, 464 (1988); (b) D. M. Bishop and J. Pipin, J. Chem. Phys. 91, 3549 (1989).
[15] W-C. Liu, Phys. Rev. A 56, 4938 (1998).

[16] Th. Mercouris and C. A. Nicolaides, J. Phys. B 21, L285 (1988).

[17] C. A. Nicolaides and Th. Mercouris, Chem. Phys. Lett. 159, 45 (1989).

[18] Th. Mercouris and C. A. Nicolaides, J. Phys. B 23, 2037 (1990); Phys. Rev. A 45, 2116 (1992).

[19] C. A. Nicolaides, Th. Mercouris, and G. Aspromallis, J. Opt. Soc. Am. B 7, 494 (1990).

[20] C. A. Nicolaides, Th. Mercouris, and N. Piangos, J. Phys. B 23, L669 (1990).

[21] Th. Mercouris, Y. Komninos, S. Dionissopoulou, and C. A. Nicolaides, Phys. Rev. A 50, 4109 (1994).

[22] Th. Mercouris and C. A. Nicolaides, J. Phys. B 24, L57 (1991).

[23] C. A. Nicolaides and Th. Mercouris, Phys. Rev. A 44, 7827 (1991).

[24] Th. Mercouris and C. A. Nicolaides, J. Phys. B 24, L557 (1991).

[25] M. Bylicki, S. I. Themelis, and C. A. Nicolaides, J. Phys. B 27, 2741 (1994).

[26] C. Haritos, Th. Mercouris, and C. A. Nicolaides, J. Phys. B 31, L783 (1998); C. A. Nicolaides, C. Haritos, and Th. Mercouris, Phys. Rev. A 55, 2830 (1997).

[27] J. Purvis, M. Dörr, M. Terao-Dunseath, C. J. Joachain, P. G. Burke, and C. J. Noble, Phys. Rev. Lett. 71, 3943 (1993). 
[28] M. Dörr, J. Purvis, M. Terao-Dunseath, P. G. Burke, C. J. Joachain, and C. J. Noble, J. Phys. B 28, 4481 (1995).

[29] D. H. Glass, P. G. Burke, C. J. Noble, and G. B. Wöste, J. Phys. B 31, L667 (1998).

[30] (a) M. Kutzner, M. Felton, and D. Winn, Phys. Rev. A 45, 7761 (1992); (b) J. Pipin and D. M. Bishop, J. Phys. B 25, 17 (1992).

[31] C. A. Nicolaides and S. I. Themelis, J. Phys. B 26, 2217 (1993).

[32] M. Jaszunski and B. O. Roos, Mol. Phys. 52, 1209 (1984).

[33] E. F. Archibong and A. J. Thakkar, Chem. Phys. Lett. 173, 579 (1990).

[34] S. P. A. Sauer, J. Phys. B 30, 3773 (1997).

[35] S. I. Themelis and C. A. Nicolaides, Phys. Rev. A 46, R21 (1992)

[36] C. A. Nicolaides and S. I. Themelis, J. Phys. B 26, L387 (1993).

[37] S. I. Themelis and C. A. Nicolaides, Phys. Rev. A 52, 2439 (1995).

[38] S. I. Themelis and C. A. Nicolaides, Phys. Rev. A 59, 2500 (1999).

[39] J. H. Shirley, Phys. Rev. B 138, B979 (1965).

[40] S-I. Chu and W. P. Reinhardt, Phys. Rev. Lett. 39, 1195 (1977).

[41] A. Maquet, S.-I. Chu, and W. P. Reinhardt, Phys. Rev. A 27, 2946 (1983).

[42] W. P. Reinhardt, Int. J. Quantum Chem., Symp. 10, 359 (1976).

[43] C. Cerjan, R. Hedges, C. Holt, W. P. Reinhardt, K. Scheiber, and J. J. Wendoloski, Int. J. Quantum Chem. 14, 393 (1978).
[44] C. D. Doolen, J. Nuttall, and R. W. Stagat, Phys. Rev. A 10, 1613 (1974).

[45] C. D. Doolen, J. Phys. B 8, 525 (1975).

[46] J. Aguilar and J. M. Combes, Commun. Math. Phys. 22, 269 (1972); E. Balslev and J. M. Combes, ibid. 22, 280 (1972).

[47] C. A. Nicolaides and S. I. Themelis, Phys. Rev. A 45, 349 (1992).

[48] S.-I. Chu, Adv. Chem. Phys. 73, 739 (1989).

[49] R. M. Potvliege and R. Shakeshaft, in Atoms in Intense Laser Fields (Ref. [6]), p. 373.

[50] G. J. Pert, IEEE J. Quantum Electron. QE-8, 623 (1972).

[51] L. Armstrong, B. L. Beers, and S. Feneuille, Phys. Rev. A 12, 1903 (1975).

[52] C. A. Nicolaides and D. R. Beck, Phys. Lett. 65A, 11 (1978).

[53] C. A. Nicolaides and D. R. Beck, Int. J. Quantum Chem. 14, 457 (1978).

[54] C. A. Nicolaides, Y. Komminos, and Th. Mercouris, Int. J. Quantum Chem., Symp. 15, 355 (1981).

[55] A. M. Dykhne and A. V. Chaplik, Zh. Eksp. Teor. Fiz. 40, 1427 (1961) [Sov. Phys. JETP 13, 1002 (1961)].

[56] C. A. Nicolaides, in Advanced Theories and Computational Approaches to the Electronic Structures of Molecules, edited by C. E. Dykstra (Reidel, Dordrecht, 1984), p. 161; Int. J. Quantum Chem. 60, 119 (1996).

[57] S. H. Autler and C. H. Townes, Phys. Rev. 100, 703 (1955).

[58] A. D. Buckingham, Adv. Chem. Phys. 12, 107 (1967).

[59] C. Froese-Fischer, Comput. Phys. Commun. 14, 145 (1978).

[60] S. I. Themelis and C. A. Nicolaides, Phys. Rev. A 49, 3089 (1994).

[61] U. V. Ammosov, N. B. Delone, and V. P. Krainov, Zh. Eksp. Teor. Fiz. 91, 2008 (1986) [Sov. Phys. JETP 64, 1191 (1986)]. 